\title{
Desenvolvimento de laranjeiras variedade "de Russas" pés francos e clones enxertados no semiárido Cearense
}

\author{
Kassio Ewerton Santos SOMBRA ${ }^{1 *}$, Henrique Maia de ANDRADE ${ }^{2}$, Francisco Leandro Costa \\ LOUREIRO $^{3}$, Francisco Edilmar de OLIVEIRA ${ }^{2}$, Debora Costa BASTOS ${ }^{4}$, \\ Cleilson do Nascimento UCHÔA ${ }^{2}$
}

\footnotetext{
${ }^{1}$ Programa de Pós-Graduação em Agronomia (Fitotecnia/Fitossanidade), Universidade Federal do Ceará, Fortaleza, CE, Brasil. ${ }^{2}$ Instituto Federal de Educação, Ciência e Tecnologia do Ceará, Campus Limoeiro do Norte, Limoeiro de Norte, CE, Brasil.

${ }^{3}$ Programa de Pós-Graduação em Fitotecnia, Universidade Federal Rural do Semi-Árido, Mossoró, RN, Brasil.

${ }^{4}$ Empresa Brasileira de Pesquisa Agropecuária, Embrapa Semiárido, Petrolina, PE, Brasil.

*E-mail: kassiosombra@gmail.com
}

Recebido em janeiro/2018; Aceito em julho/2018.

\begin{abstract}
RESUMO: A laranja 'de Russas' é uma variedade tradicional adaptada ao semiárido com potencial de atender o mercado de frutas de mesa. O trabalho avaliou o desenvolvimento de laranjeiras pés francos na fase vegetativa, comparando-as com clones BRS Russas sobre porta-enxertos, e na fase reprodutiva, utilizando quatro intervalos de idade para determinar a curva de crescimento. Analisaram-se a altura (H); diâmetros (DL e DR) e volume médio da copa $\left(\mathrm{V}^{3}\right)$; diâmetro do caule (D) e razão de compatibilidade copa/porta-enxerto (IC). As laranjeiras pés francos apresentaram desenvolvimento vegetativo inferior ao dos clones BRS Russas na fase juvenil, indicando que a ausência da juvenilidade proporcionada pelo uso de mudas enxertadas, entre outros fatores, como a alta afinidade entre os clones e os porta-enxertos, pode ter favorecido a formação das copas dos clones, destacando-se o desenvolvimento do clone BRS Russas 04 sobre os citrandarins ‘Indio e San Diego' diante dos estresses abióticos típicos do semiárido cearense. As laranjeiras pés francos em período produtivo apresentavam alto vigor, com predomínio de crescimento vertical, apesar de ganharem em diâmetro e volume de copa à medida que envelhecem e estabilizam a produção, permitindo a obtenção de produtividades mais elevadas e retorno econômico significativo na citricultura tradicional.
\end{abstract}

Palavras-chave: agricultura familiar, citros, diversificação, melhoramento genético.

\section{Development of orange trees variety 'Russas' feet francs and clones grafted in the semiarid of Ceará, Brazil}

\begin{abstract}
The 'Russas' orange is a traditional variety adapted to the semiarid region with the potential to meet the fruit market. The study evaluated the development of 'Russas' orange feet francs in the vegetative stage, comparing them with clones BRS Russas on different rootstocks, and the reproductive stage, using four age intervals to determine the growth curve. We analyzed the height $(\mathrm{H})$; diameters (DL and DR) and average volume of the canopy $\left(\mathrm{V}^{3}\right)$; stem diameter (D) and reason of compatibility canopy/rootstock (IC). The orange feet francs presented below the vegetative development of the BRS Russas clones in juvenile phase, indicating that the absence of juvenility afforded by the use of grafted seedlings, among other factors, such as the high affinity between the clones and rootstocks, may have favored the formation of the canopies of the clones, highlighting the development of the BRS Russas 04 clone on the 'Indio and San Diego' citrandarins before the abiotic typical of semiarid. The orange feet francs in the productive period presented high force, with predominance of vertical growth, despite the gain in diameter and canopy volume as they grow older and stabilize production, allowing you to obtain higher yields and significant economic return in citriculture traditional.
\end{abstract}

Keywords: family farming, citrus, diversification, genetic improvement.

\section{INTRODUÇÃO}

Os citros estão entre as espécies mais cultivadas mundialmente, principalmente para consumo 'in natura' ou formas processadas e industrializadas, destacando-se o suco de laranja, um dos principais commodities mundiais, entre outros subprodutos de alto valor agregado (CUNHA SOBRINHO et al., 2013; SILVA et al., 2016; PASSOS et al., 2016). O Brasil detém o título de maior produtor mundial de laranja (Citrus sinensis L. Osbeck) (Rutaceae), além de, maior produtor e exportador de suco de laranja concentrado congelado (FCOJ), consolidando-se no cenário citrícola mundial (IBGE, 2017;
USDA, 2017a). A produção brasileira de laranja deve crescer 34\% na safra 2017/2018 (julho a junho), em relação à safra anterior (considerada a menor dos últimos 25 anos), conforme relatório do Departamento de Agricultura dos Estados Unidos (USDA, 2017b), atribuindo-se o aumento as condições climáticas favoráveis e plantas com maior quantidade de energia, resultando em maior tamanho das frutas cultivadas na área comercial de São Paulo e no oeste de Minas Gerais, impulsionando assim a produção aos 471 milhões de caixas de $40,8 \mathrm{~kg}$. Os números expressivos incidem diretamente no mercado de suco de laranja concentrado congelado (FCOJ), 
tornando o Brasil responsável por cerca de $61,3 \%$ da produção e 75,3\% das exportações mundiais (USDA, 2017b).

A região Nordeste, segunda maior produtora de laranja do país, responde por cerca de $11,3 \%$ da produção nacional, com 1.658.588 toneladas de frutas e rendimento médio de 13,67

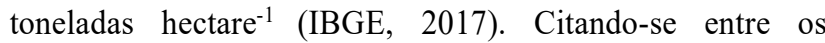
principais desafios para a expansão da citricultura na região: o clima seco e quente, típico de semiárido, predominante na maior extensão territorial; seleção e obtenção de cultivares adaptadas aos estresses bióticos e abióticos; aquisição de mudas com garantia de qualidade (genética e fitossanitária); solos de boa fertilidade, ausente de camadas compactadas, impedimentos físicos, com resistência a penetração ou problemas de má drenagem em períodos de chuva intensa; água para fins de irrigação sem contaminação por sais ou metais; assistência técnica e extensão rural pública e de qualidade, assim como, incentivo ao associativismo e acesso a programas de crédito rural para aquisição de insumos e equipamentos para potencialização da produção citrícola (SOMBRA et al., 2016; SOMBRA et al., 2018a).

Porém, apesar dos possíveis desafios a serem enfrentados pela citricultura na região Nordeste, o estado do Ceará mostrase promissor para a revitalização e expansão da cadeia produtiva, destacando-se por apresentar situação fitossanitária sob controle, com monitoramento ou ausência de principais pragas e doenças de importância econômica como, por exemplo: a mosca-negra (Aleurocanthus woglumi Ashby.) (Hemiptera: Aleyrodidae) e o psilídeo (Diaphorina citri Kuwayama) (Hemiptera: Liviidae), inseto vetor das bactérias (Candidatus Liberibacter spp.) que causam o Greening (Huanglongbing/HLB); assim como, doenças chave na citricultura mundial, como o cancro cítrico (Xanthomonas axonopodis pv. citri), e a pinta preta (Guignardia citricarpa Kiely) (SOMBRA et al., 2018a). Uma possível expansão da citricultura no Ceará também se beneficiaria pelo potencial dos perímetros públicos irrigados, um total de 14 no estado, além de contar com a existência de uma variedade tradicional de laranja doce (C. sinensis), a laranja "de Russas", adaptada ao semiárido e com potencial de satisfazer a oferta nacional de fruta de mesa (PASSOS et al., 2013; SOMBRA et al., 2016)

Diante da existência da laranja 'de Russas', a Empresa Brasileira de Pesquisa Agropecuária (EMBRAPA) desenvolveu pesquisas de identificação, seleção e clonagem de laranjeiras (matrizes) com características e frutos de qualidade diferenciada, lançados nacionalmente, conforme Passos et al., (2013).

A laranja 'de Russas, segundo Passos et al., (2013), é resultado da introdução de sementes de laranja doce por jesuítas portugueses logo após o descobrimento, no século XVI, sendo cultivada e selecionada empiricamente através de figuras religiosas e agricultores familiares, até culminar com a expansão da citricultura no começo do século XX, quando era comercializada nos mercados locais e agências de ônibus, onde rotineiramente ouvia-se a propaganda dos pequenos vendedores - Olha a laranja 'De Russas'! - Oferecendo os frutos cítricos aos viajantes e passageiros das caravanas (PASSOS et al., 2013; SOMBRA et al., 2016).

Considerando a importância social e econômica desta variedade de laranja para os produtores do Ceará, e do semiárido nordestino, objetivou-se com este trabalho avaliar o desenvolvimento de plantas propagadas via semente (pé franco) da variedade tradicional laranja 'de Russas', nas fases vegetativa e reprodutiva (produtiva), comparando-as com clones BRS Russas enxertados sobre diferentes portaenxertos.

\section{MATERIAL E MÉTODOS}

2.1. Caracterização da área de estudo

$\mathrm{O}$ trabalho foi desenvolvido entre os meses de janeiro e outubro de 2017 em pomares cítricos produtivos e experimentais localizados nas zonas rurais dos municípios de Limoeiro do Norte, Quixeré e Russas, no Baixo Jaguaribe, Ceará. O clima da microrregião é classificado como seco e muito quente, do tipo BSw'h' (Köppen) (ALVARES et al., 2014), localizando-se no semiárido cearense, e inserindo-se no bioma caatinga. A temperatura média anual é de aproximadamente $27,1^{\circ} \mathrm{C}$, oscilando entre $22,3^{\circ} \mathrm{C}$ e $34,8^{\circ} \mathrm{C}$, com umidade relativa do ar variando de $44 \%$ a $85 \%$, apresentando regime pluviométrico médio de $857,7 \mathrm{~mm}$, variando entre 548 e $992 \mathrm{~mm}$, com chuvas irregulares distribuídas ao longo do ano, e duas estações bem definidas, uma seca que vai geralmente de junho a janeiro, e uma chuvosa, de fevereiro a maio (verão e outono).

Os solos no Baixo Jaguaribe são predominantemente 'Neossolos Flúvicos (RY)', definidos como solos minerais não hidromórficos, com alto potencial agrícola quando apresentam textura média e boa drenagem. A vegetação nativa é formada principalmente por carnaubeiras (Copernicia prunifera Mill. H. E. Moore) (Arecaceae), intercaladas por pequenas e médias propriedades conduzidas em sua maioria sob preceitos da agricultura familiar (SOMBRA et al., 2016; SOMBRA et al., 2018a).

\subsection{Análise comparativa do desenvolvimento vegetativo}

A caracterização do desenvolvimento vegetativo foi dividida entre fase juvenil (vegetativa) e reprodutiva (produtiva), comparando-se as plantas 'pé franco' (propagadas por semente) da variedade tradicional 'laranja de Russas', com clones BRS Russas, identificados na região, melhorados e disponibilizados pela Embrapa (PASSOS et al., 2013), sobre diferentes porta-enxertos.

Primeiramente, caracterizaram-se as laranjeiras 'de Russas' pés francos no período juvenil, com idade média de 30 meses, comparando-as com diferentes combinações entre clones 'BRS Russas' e porta-enxertos, tolerantes ou resistentes aos principais estresses bióticos e abióticos, que acometem a citricultura em condições semiáridas. Posteriormente, avaliaram-se laranjeiras 'de Russas' pés francos em diferentes períodos da fase reprodutiva (produtiva), buscando-se determinar a curva de desenvolvimento das plantas cítricas propagadas via semente pelos citricultores da região.

As laranjeiras 'de Russas', na fase juvenil e reprodutiva, localizavam-se em pomares particulares, inseridos na agricultura familiar dos municípios, enquanto os clones 'BRS Russas' localizavam-se na Área Experimental LB Carpina (Figura 1), implantada pela Empresa Brasileira de Pesquisa Agropecuária (Embrapa) e acompanhada pelo Núcleo de Pesquisa em Citros (NPCitrus/IFCE).

\subsection{Delineamento experimental e tratamentos}

Adotou-se delineamento inteiramente casualizado (DIC), dividindo-se a analise entre fase juvenil e reprodutiva. Na fase juvenil utilizaram-se laranjeiras 'pés francos' como controle, comparando-as com combinações entre 08 clones BRS Russas enxertados sobre diferentes porta-enxertos, num total de 20 tratamentos com quatro repetições cada (80 plantas úteis). 
Listando-se os clones BRS Russas 01 a 08 como copas, e os citrandarins 'Indio, Riverside e San Diego', além do híbrido 069, como porta-enxertos avaliados. Na fase reprodutiva, adotaram-se como tratamentos quatro diferentes intervalos de idade de laranjeiras 'de Russas' (pés francos), listando-se: laranjeiras com até 10 anos de idade; laranjeiras com idade entre 11 e 20 anos; laranjeiras com idade entre 21 e 30 anos; e laranjeiras com idade superior a 30 anos.

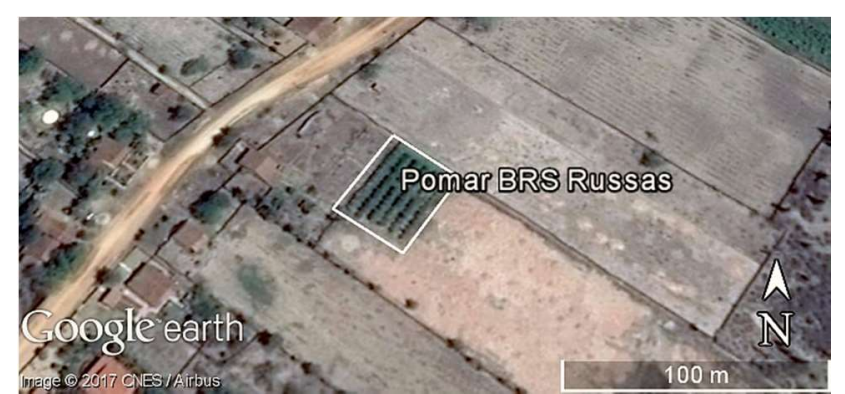

Figura 1. Área experimental LB Carpina - Pomar BRS Russas $\left(5^{\circ} 0^{\prime} 48.45^{\prime \prime} \mathrm{S} ; 38^{\circ} 2^{\prime} 1.60^{\prime} \mathrm{O}\right)$, Russas-CE-Brasil. Fonte: Google Earth Pro 7.1.5. Data da imagem: 29/12/2016.

Figure 1. Experimental area LB Carpina - Russas BRS Orchard $\left(5^{\circ} 0^{\prime} 48.45^{\prime \prime} \mathrm{S} ; 38^{\circ} 2^{\prime} 1.60^{\prime \prime} \mathrm{W}\right)$, Russas-CE-Brazil.

\subsection{Variáveis analisadas}

Analisaram-se as alturas $(\mathrm{H})$, medida do colo ao ápice; diâmetro da copa nos sentidos entre plantas e entre linhas (DL e DR), utilizando-se os dados para cálculo do volume médio da copa $\left(\mathrm{V}^{3}\right)$ através da aplicação da fórmula descrita por Fallahi; Rodney (1992):

$$
\mathrm{V}=(\pi / 6) \times \mathrm{H} \times \mathrm{Dl} \times \mathrm{Dr}
$$

(Equação 1)

Nas combinações copa e porta-enxerto, mensurou-se também o diâmetro do caule, $5 \mathrm{~cm}$ abaixo $(\mathrm{DC} \downarrow)$ e acima (DC $\uparrow)$ do ponto de enxertia, calculando-se a razão de compatibilidade (IC) entre o porta-enxerto e enxerto, e tomando-se o valor 1 como equivalente da compatibilidade plena (RODRIGUES et al., 2015; RODRIGUES et al., 2016; LOUREIRO et al., 2016; SOMBRA et al., 2018b), enquanto no tratamento controle, com laranjeiras 'de Russas' pés francos, o diâmetro do caule (DC) foi mensurado a altura média de $50 \mathrm{~cm}$ do colo das plantas.

\subsection{Análise estatística}

Realizou-se a análise de regressão para ao volume de copa $\left(\mathrm{V}^{3}\right)$ em função do número de dias após o transplantio ou semeadura. Os dados foram submetidos à análise de variância (ANOVA), e nos casos de diferença significativa, compararam-se as médias aplicando-se o Teste de Scott-Knott ao nível de $5 \%$ de probabilidade $(\mathrm{p} \leq 0,05)$, com auxílio do software ASSISTAT ${ }^{\circledR}$ (SILVA; AZEVEDO, 2016).

\section{RESULTADOS}

Constatou-se diferença significativa em todas as variáveis do desenvolvimento vegetativo, resumindo-se os resultados aos 30 meses após o transplantio nas Tabelas 1 e 2, respectivamente. A análise dividiu-se nas três principais variáveis, associando a altura $(\mathrm{H} / \mathrm{m})$ ao diâmetro de copa $(\mathrm{D} / \mathrm{m})$ para cálculo do volume médio de copa $\left(\mathrm{V}^{3} / \mathrm{m}^{3}\right)$, constatando-se que a laranjeira de Russas 'pé franco', diferiu estatisticamente da maioria dos tratamentos com clones BRS Russas, apresentando desenvolvimento vegetativo inferior em todas as variáveis, como altura média $(\mathrm{H})$ de 2,33m; diâmetro médio de copa $\left(\mathrm{D}_{\text {médio }}\right)$ de $1,486 \mathrm{~m}$ e volume médio de copa $\left(\mathrm{V}^{3}\right)$ de $2,7248 \mathrm{~m}^{3}$ (Tabela 2).

Tabela 1. Altura $(\mathrm{H} / \mathrm{m})$ e diâmetro médio de copa $\left(\mathrm{D}_{\text {médio }}\right)$ de 'pés francos' de 'laranja de Russas' e clones BRS Russas sobre portaenxertos, aos 30 meses após o transplantio, Russas, Ceará.

Table 1. Height $(\mathrm{H} / \mathrm{m})$ and mean crown diameter (DM) of 'free standing' of 'Russas orange' and Russas BRS clones on rootstocks, at 30 months after transplanting, Russas, Ceará.

\begin{tabular}{|c|c|c|c|}
\hline \multicolumn{2}{|c|}{ Tratamentos } & $\mathrm{H}(\mathrm{m})$ & $\mathrm{D}_{\text {médio }}(\mathrm{m})$ \\
\hline \multicolumn{2}{|c|}{ Controle (pé franco) } & $2,33 \pm 0,07 \mathrm{c}$ & $1,486 \pm 0,13 \mathrm{~d}$ \\
\hline Copa & Porta-enxerto & - & - \\
\hline \multirow{4}{*}{ BRS Russas 01} & C. Indio & $2,42 \pm 0,14 \mathrm{c}$ & $2,471 \pm 0,45 \mathrm{~b}$ \\
\hline & C. Diego & $2,41 \pm 0,14 \mathrm{c}$ & $2,631 \pm 0,23 \mathrm{~b}$ \\
\hline & C. Swingle & $2,49 \pm 0,10 \mathrm{c}$ & $2,544 \pm 0,15 \mathrm{~b}$ \\
\hline & C. Indio & $2,16 \pm 0,36 \mathrm{~d}$ & $2,136 \pm 0,52 \mathrm{c}$ \\
\hline \multirow[t]{2}{*}{ BRS Russas 02} & C. Diego & $2,49 \pm 0,03 \mathrm{c}$ & $2,556 \pm 0,14 \mathrm{~b}$ \\
\hline & C. Swingle & $2,38 \pm 0,09 \mathrm{c}$ & $2,964 \pm 0,19 \mathrm{a}$ \\
\hline \multirow{3}{*}{ BRS Russas 03} & C. Diego & $2,60 \pm 0,11 \mathrm{~b}$ & $2,829 \pm 0,24 \mathrm{a}$ \\
\hline & C. Swingle & $2,46 \pm 0,12 \mathrm{c}$ & $2,891 \pm 0,18 \mathrm{a}$ \\
\hline & C. Indio & $3,32 \pm 0,16 \mathrm{a}$ & $3,104 \pm 0,14 \mathrm{a}$ \\
\hline \multirow[t]{2}{*}{ BRS Russas 04} & C. Diego & $3,16 \pm 0,06 \mathrm{a}$ & $3,319 \pm 0,26 \mathrm{a}$ \\
\hline & C. Swingle & $3,19 \pm 0,08 \mathrm{a}$ & $3,112 \pm 0,21 \mathrm{a}$ \\
\hline \multirow[t]{2}{*}{ BRS Russas 05} & C. Indio & $2,96 \pm 0,40 \mathrm{~b}$ & $2,986 \pm 0,35 \mathrm{a}$ \\
\hline & C. Indio & $2,92 \pm 0,28 \mathrm{~b}$ & $2,959 \pm 0,19 \mathrm{a}$ \\
\hline \multirow[t]{2}{*}{ BRS Russas 06} & C. Diego & $2,75 \pm 0,25 \mathrm{~b}$ & $2,970 \pm 0,23 \mathrm{a}$ \\
\hline & C. Swingle & $2,61 \pm 0,16 \mathrm{~b}$ & $2,685 \pm 0,39 \mathrm{~b}$ \\
\hline \multirow{2}{*}{ BRS Russas 07} & C. Swingle & $2,82 \pm 0,16 \mathrm{~b}$ & $2,972 \pm 0,26 \mathrm{a}$ \\
\hline & Híbrido 069 & $2,34 \pm 0,23 \mathrm{c}$ & $1,949 \pm 0,15 \mathrm{c}$ \\
\hline \multirow{2}{*}{ BRS Russas 08} & C. Swingle & $2,67 \pm 0,19 \mathrm{~b}$ & $2,854 \pm 0,08 \mathrm{a}$ \\
\hline & Híbrido 069 & $2,41 \pm 0,42 \mathrm{c}$ & $2,459 \pm 0,32 \mathrm{~b}$ \\
\hline \multirow{2}{*}{\multicolumn{2}{|c|}{$\begin{array}{l}\text { C. V. (\%) } \\
\text { Valor F }\end{array}$}} & 11,11 & 13,25 \\
\hline & & $11,2312 * *$ & $11,3626^{* *}$ \\
\hline
\end{tabular}

As médias seguidas pela mesma letra não diferem estatisticamente entre si. ${ }^{* *}$ significativo ao nível de $1 \%$ de probabilidade (Scott-Knott, $\mathrm{p}<0.01$ ).

Tabela 2. Volume médio de copa $\left(\mathrm{V}^{3} / \mathrm{m}^{3}\right)$ de 'pés francos' de 'laranja de Russas' e clones BRS Russas sobre porta-enxertos, aos 30 meses após o transplantio, Russas, Ceará.

Table 2. Average cup volume $\left(\mathrm{V}^{3} / \mathrm{m}^{3}\right)$ of 'free standing' of 'Russas orange' and Russas BRS clones on rootstocks, at 30 months after transplanting, Russas, Ceará.

\begin{tabular}{cll}
\hline \multicolumn{2}{c}{ Tratamentos } & $\mathrm{V}^{3}\left(\mathrm{~m}^{3}\right)$ \\
\hline \multicolumn{2}{c}{ Controle (pé franco) } & $2,7248 \pm 0,510 \mathrm{~d}$ \\
\hline Copa & Porta-enxerto & - \\
\hline \multirow{2}{*}{ BRS Russas 01 } & C. Indio & $8,2708 \pm 2,747 \mathrm{c}$ \\
& C. Diego & $8,9020 \pm 0,233 \mathrm{c}$ \\
& C. Swingle & $8,5227 \pm 1,860 \mathrm{c}$ \\
BRS Russas 02 & C. Indio & $5,9171 \pm 3,424 \mathrm{c}$ \\
& C. Diego & $8,5484 \pm 0,983 \mathrm{c}$ \\
& C. Swingle & $11,0860 \pm 1,868 \mathrm{~b}$ \\
BRS Russas 03 & C. Diego & $10,9424 \pm 1,510 \mathrm{~b}$ \\
& C. Swingle & $10,8650 \pm 1,578 \mathrm{~b}$ \\
BRS Russas 04 & C. Indio & $16,9089 \pm 2,361 \mathrm{a}$ \\
& C. Diego & $18,4455 \pm 3,248 \mathrm{a}$ \\
BRS Russas 05 & C. Swingle & $16,3056 \pm 2,254 \mathrm{a}$ \\
& C. Indio & $14,7319 \pm 5,930 \mathrm{a}$ \\
BRS Russas 06 & C. Indio & $13,6658 \pm 2,842 \mathrm{~b}$ \\
& C. Diego & $12,9741 \pm 3,138 \mathrm{~b}$ \\
BRS Russas 07 & C. Swingle & $10,2860 \pm 3,499 \mathrm{~b}$ \\
& C. Swingle & $13,2401 \pm 2,817 \mathrm{~b}$ \\
BRS Russas 08 & Híbrido 069 & $4,7937 \pm 1,210 \mathrm{~d}$ \\
& C. Swingle & $11,4231 \pm 0,985 \mathrm{~b}$ \\
& Híbrido 069 & $8,0176 \pm 2,360 \mathrm{c}$ \\
\hline C. V. (\%) & & 34,31 \\
Valor F & & $10,4848 * *$ \\
\hline
\end{tabular}

As médias seguidas pela mesma letra não diferem estatisticamente entre si. ** significativo ao nível de $1 \%$ de probabilidade (Scott-Knott, $\mathrm{p}<0.01$ ). 
Os maiores resultados foram obtidos pelo clone BRS Russas 04 sobre citrandarin 'Indio' (com altura de 3,32m e diâmetro médio de copa de $3,104 \mathrm{~m}$, resultando num volume de $16,9089 \mathrm{~m}^{3}$ de copa); citrandarin 'San Diego' (3,16m de altura, diâmetro médio de $3,319 \mathrm{~m}$ e volume médio de copa de $18,4455 \mathrm{~m}^{3}$ ); e citrumelo 'Swingle' (com $3,19 \mathrm{~m}$ e $3,112 \mathrm{~m}$, de altura e diâmetro, respectivamente, e $16,3056 \mathrm{~m}^{3}$ de volume médio de copa).

Os clones BRS Russas mantiveram a predominância sobre as laranjeiras 'de Russas' pés francos ao analisar os diâmetros dos caules, destacando-se por exemplo, resultados do clone BRS Russas 02 sobre citrandarin 'San Diego', com diâmetros de $124,8 \mathrm{~mm}$ abaixo (DC $\downarrow$ ), e $115,42 \mathrm{~mm}$ acima (DC $\uparrow$ ) do ponto de enxertia, e razão de compatibilidade (IC) de 0,922; do clone BRS Russas 04 sobre citrandarin 'Indio', com $120,22 \mathrm{~mm}$ e 108,05, abaixo e acima, e IC de 0,901; e do mesmo clone sobre citrandarin 'San Diego', com 116,55mm e 103,52mm, e IC de 0,889; e por último, do clone BRS Russas 06 sobre citrandarin 'Indio', com 126,57mm e 118,75, apresentando IC de 0,938 (Tabela 3).

Tabela 3. Diâmetro do caule, $5 \mathrm{~cm}$ abaixo (DC $\downarrow$ ) e acima (DC $\uparrow$ ) do ponto de enxertia, e a razão de compatibilidade (IC) de 'pés francos' de 'laranja de Russas' e clones BRS Russas sobre porta-enxertos, aos 30 meses após o transplantio, Russas, Ceará.

Table 3. Diameter of the stem, $5 \mathrm{~cm}$ below (DC $\downarrow$ ) and above (DC $\uparrow$ ) of the grafting point, and the 'free feet' compatibility ratio (IC) of 'Russas orange' and Russas BRS clones on rootstocks, 30 months after transplanting, Russas, Ceará.

\begin{tabular}{|c|c|c|c|c|}
\hline \multicolumn{2}{|c|}{ Tratamentos } & \multicolumn{2}{|c|}{$(\mathrm{mm})$} & $\mathrm{IC}$ \\
\hline \multicolumn{2}{|c|}{ Controle (pé franco) } & \multicolumn{2}{|c|}{47,05} & - \\
\hline Copa & Porta-enxerto & - & - & \\
\hline \multirow{3}{*}{$\begin{array}{c}\text { BRS Russas } \\
01\end{array}$} & C. Indio & $91,52 \mathrm{~b}$ & $78,55 \mathrm{~b}$ & $0,869 \mathrm{a}$ \\
\hline & C. Diego & $98,97 \mathrm{~b}$ & $73,77 \mathrm{c}$ & $0,749 \mathrm{~b}$ \\
\hline & C. Swingle & $99,15 \mathrm{~b}$ & $85,60 \mathrm{~b}$ & $0,865 \mathrm{a}$ \\
\hline \multirow{3}{*}{$\begin{array}{c}\text { BRS Russas } \\
02\end{array}$} & C. Indio & $82,12 \mathrm{c}$ & $68,02 \mathrm{c}$ & $0,822 \mathrm{a}$ \\
\hline & C. Diego & $124,80 \mathrm{a}$ & $115,42 \mathrm{a}$ & $0,922 \mathrm{a}$ \\
\hline & C. Swingle & $98,47 \mathrm{~b}$ & $90,20 \mathrm{~b}$ & $0,918 \mathrm{a}$ \\
\hline BRS Russas & C. Diego & $137,65 \mathrm{a}$ & $100,70 \mathrm{a}$ & $0,732 \mathrm{~b}$ \\
\hline 03 & C. Swingle & $104,32 b$ & $88,62 \mathrm{~b}$ & $0,850 \mathrm{a}$ \\
\hline \multirow{3}{*}{$\begin{array}{c}\text { BRS Russas } \\
04\end{array}$} & C. Indio & $120,22 \mathrm{a}$ & $108,05 \mathrm{a}$ & $0,901 \mathrm{a}$ \\
\hline & C. Diego & $116,55 \mathrm{a}$ & $103,51 \mathrm{a}$ & $0,889 \mathrm{a}$ \\
\hline & C. Swingle & $108,60 \mathrm{~b}$ & $92,62 \mathrm{~b}$ & $0,847 \mathrm{a}$ \\
\hline $\begin{array}{c}\text { BRS Russas } \\
05\end{array}$ & C. Indio & $123,57 \mathrm{a}$ & $92,50 \mathrm{~b}$ & $0,753 \mathrm{~b}$ \\
\hline \multirow{3}{*}{$\begin{array}{c}\text { BRS Russas } \\
06\end{array}$} & C. Indio & $126,57 \mathrm{a}$ & $118,75 \mathrm{a}$ & $0,938 \mathrm{a}$ \\
\hline & C. Diego & $128,52 \mathrm{a}$ & $112,15 \mathrm{a}$ & $0,870 \mathrm{a}$ \\
\hline & C. Swingle & $103,39 \mathrm{~b}$ & $98,22 \mathrm{a}$ & $0,948 \mathrm{a}$ \\
\hline BRS Russas & C. Swingle & $94,82 \mathrm{~b}$ & $79,60 \mathrm{~b}$ & $0,825 \mathrm{a}$ \\
\hline 07 & Híbrido 069 & $98,10 \mathrm{~b}$ & $66,32 \mathrm{c}$ & $0,673 \mathrm{~b}$ \\
\hline BRS Russas & C. Swingle & $96,82 \mathrm{~b}$ & $83,65 \mathrm{~b}$ & $0,863 \mathrm{a}$ \\
\hline 08 & Híbrido 069 & $97,40 \mathrm{~b}$ & $88,12 \mathrm{~b}$ & $0,906 \mathrm{a}$ \\
\hline C. V. (\%) & & 17,59 & 20,16 & 9,79 \\
\hline Valor F & & $10,28 * *$ & $9,90 * *$ & $3,33 * *$ \\
\hline
\end{tabular}

As médias seguidas pela mesma letra não diferem estatisticamente entre si. ** significativo ao nível de $1 \%$ de probabilidade (Scott-Knott, $\mathrm{p}<0.01$ ).

Os resultados foram superiores aos registrados na literatura para outras variedades e espécies cítricas, como constata-se através de Loureiro et al., (2016), avaliando pomeleiro 'Star Ruby' sobre porta-enxertos, obtiveram valores significativamente inferiores em todas as variáveis e tratamentos, assim como, Rodrigues et al., (2016), avaliaram quatro copas de citros sobre 14 diferentes porta-enxertos, e também obtiveram valores inferiores, citando-se valores de IC médio de 0,68 em combinações com a copa de laranjeira
'Pera', e valores entre 0,67 e 0,88 nas combinações com laranjeira 'Westin' e tangerineira-tangor 'Piemonte', porém, com apenas 90 dias após a transplantio (RODRIGUES et al., 2015; SOARES et al., 2015; RODRIGUES et al., 2016).

Os valores da caracterização do desenvolvimento vegetativo na fase produtiva de laranjeiras 'de Russas' pés francos, estão apresentados nas Tabelas 4 e 5.

As laranjeiras 'de Russas' pés francos em período produtivo, demonstraram desenvolvimento vigoroso, predominando o crescimento vertical durante o período de formação (0 a 10 anos), porém, ocorrendo ganho em diâmetro e volume de copa nos anos posteriores, quando as laranjeiras atingiram e estabilizaram a conformação das copas, proporcionando produtividades elevadas e permitindo a exploração econômica, conforme pode visualizar-se nas curvas de desenvolvimento vegetativo (Figuras 2 à 5).

Tabela 4. Idade média amostral, altura $(\mathrm{H})$ e volume da copa $\left(\mathrm{V}^{3}\right)$ de laranjeiras 'de Russas' (pés francos), Russas, Ceará.

Table 4. Mean age, height (H) and crown volume (V3) of 'Russas' (free-standing) orange trees, Russas, Ceará.

\begin{tabular}{cccc}
\hline $\begin{array}{c}\text { Intervalo de } \\
\text { Idade }\end{array}$ & $\begin{array}{c}\text { Idade } \\
\text { média }\end{array}$ & $\mathrm{H}(\mathrm{m})$ & $\mathrm{V}^{3}\left(\mathrm{~m}^{3}\right)$ \\
\hline$<10$ anos & $8,0 \mathrm{c}$ & $5,17 \pm 0,2 \mathrm{a}$ & $52,191 \pm 10,6 \mathrm{~b}$ \\
$\begin{array}{c}\text { Entre } 11 \mathrm{e} 20 \\
\text { anos }\end{array}$ & $13,6 \mathrm{c}$ & $6,81 \pm 1,1 \mathrm{a}$ & $104,855 \pm 20,9 \mathrm{~b}$ \\
$\begin{array}{c}\text { Entre } 21 \text { e } 30 \\
\text { anos }\end{array}$ & $22,3 \mathrm{~b}$ & $7,03 \pm 0,5 \mathrm{a}$ & $128,416 \pm 12,8 \mathrm{~b}$ \\
$\begin{array}{c}\text { Acima de } 30 \\
\text { anos }\end{array}$ & $33,3 \mathrm{a}$ & $8,80 \pm 1,1 \mathrm{a}$ & $226,544 \pm 36,0 \mathrm{a}$ \\
\hline $\begin{array}{l}\text { C. V. (\%) } \\
\text { Valor F }\end{array}$ & 33,98 & 20,76 & 26,13 \\
\hline $\begin{array}{l}\text { As médias seguidas pela mesma letra não diferem estatisticamente entre si. ** } \\
\text { (1,29* }\end{array}$
\end{tabular}

As médias seguidas pela mesma letra não diferem estatisticamente entre si. ** significativo ao nível de $1 \%$ de probabilidade (Scott-Knott, $\mathrm{p}<0.01$ ). ${ }^{\mathrm{NS}}$ não significativo.

Tabela 5. Diâmetro de caule (DC/cm), e diâmetro de copa entre planta (DL) e entre linhas (DR/m), de laranjeiras 'de Russas' (pés francos), Russas, Ceará.

Table 5. Diameter of stem $(\mathrm{DC} / \mathrm{cm})$, and crown diameter between plant (DL) and between rows (DR/m)) of 'Russas' (free-standing) orange trees, Russas, Ceará.

\begin{tabular}{cccc}
\hline $\begin{array}{c}\text { Intervalo de } \\
\text { Idade }\end{array}$ & DL $(\mathrm{m})$ & DR $(\mathrm{m})$ & $\mathrm{DC}(\mathrm{mm})$ \\
\hline$<10$ anos & $4,33 \pm 0,3 \mathrm{c}$ & $4,40 \pm 0,4 \mathrm{c}$ & $60,96 \pm 10,7 \mathrm{c}$ \\
$\begin{array}{c}\text { Entre 11 e 20 } \\
\text { anos }\end{array}$ & $6,23 \pm 1,1 \mathrm{~b}$ & $5,60 \pm 0,2 \mathrm{~b}$ & $92,31 \pm 1,5 \mathrm{~b}$ \\
$\begin{array}{c}\text { Entre 21 e } 30 \\
\text { anos }\end{array}$ & $5,73 \pm 0,5 \mathrm{~b}$ & $5,66 \pm 0,2 \mathrm{~b}$ & $99,85 \pm 4,3 \mathrm{~b}$ \\
$\begin{array}{c}\text { Acima de } 30 \\
\text { anos }\end{array}$ & $7,20 \pm 1,6 \mathrm{a}$ & $6,76 \pm 0,1 \mathrm{a}$ & $119,20 \pm 6,7 \mathrm{a}$ \\
\hline $\begin{array}{c}\text { C. V. }(\%) \\
\text { Valor F }\end{array}$ & 7,32 & 6,53 & 6,88 \\
\hline As médian & $23,1396^{* *}$ & $20,9089^{* *}$ & $42,9226^{* *}$ \\
\hline
\end{tabular}

As médias seguidas pela mesma letra não diferem estatisticamente entre si. ** significativo ao nível de $1 \%$ de probabilidade (Scott-Knott, $\mathrm{p}<0.01$ ).

\section{DISCUSSÃO}

Ao comparar os resultados com os valores obtidos por Rodrigues et al., (2016), constata-se que as combinações entre clones BRS Russas obtiveram resultados superiores em quase todas as combinações com diferentes porta-enxertos, diferindo estatisticamente das laranjeiras 'de Russas' pés francos, e apresentando alta adaptabilidade diante das condições edafoclimáticas típicas de semiárido, ressaltando-se a baixa tecnificação empregada no cultivo. 


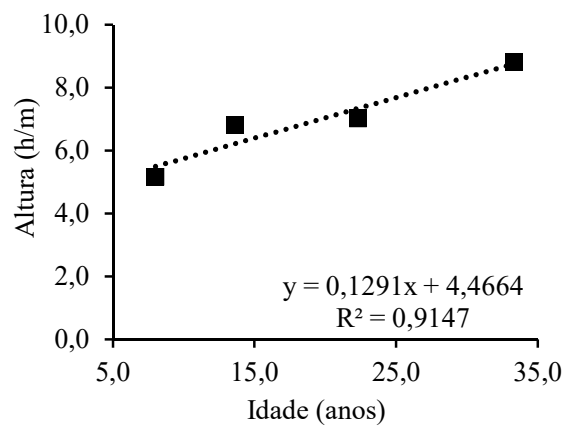

Figura 2. Altura $(\mathrm{h} / \mathrm{m})$ das laranjeiras tradicionais 'pés francos', Russas-CE-Brasil.

Figure 2. Height $(\mathrm{H} / \mathrm{m})$ of the traditional 'free-standing' orange trees, Russas-CE-Brazil

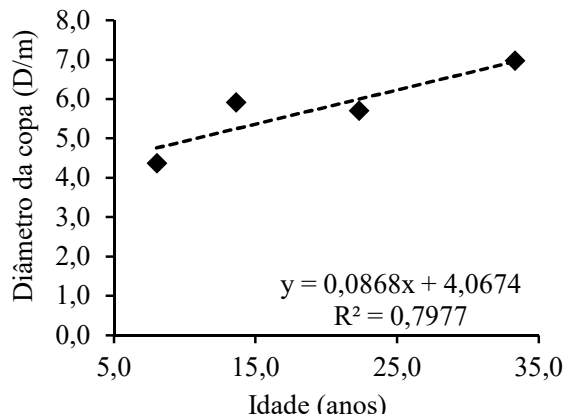

Figura 3. Diâmetro de copa $(\mathrm{D} / \mathrm{m})$ das laranjeiras 'de Russas' pés francos, Russas-CE-Brasil

Figure 3. Canopy diameter $(\mathrm{D} / \mathrm{m})$ of the Russas 'free-standing' orange trees, Russas-CE-Brazil.

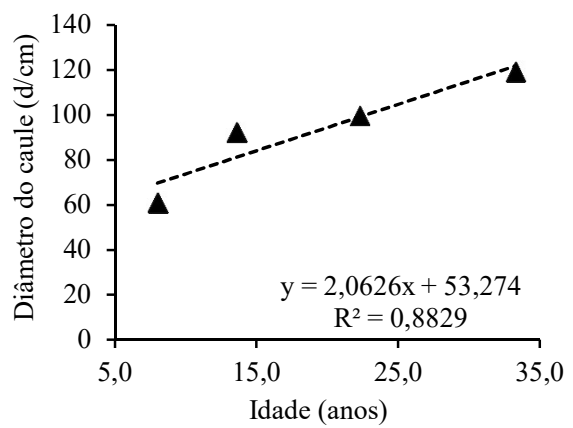

Figura 4. Diâmetro do caule (d/cm) das laranjeiras 'de Russas' 'pés francos', Russas-CE-Brasil

Figure 4. Diameter of stem $(\mathrm{d} / \mathrm{cm})$ of the Russas 'free-standing' orange trees, Russas-CE-Brazil.

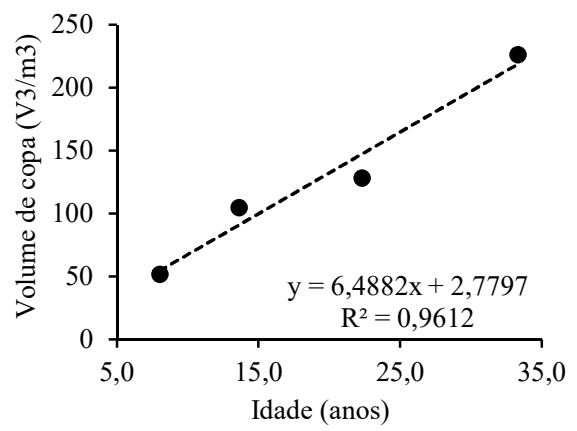

Figura 5. Volume de copa $\left(\mathrm{V}^{3} / \mathrm{m}^{3}\right)$ das laranjeiras 'de Russas' pés francos, Russas-CE-Brasil
Figure 5. Canopy volume $\left(\mathrm{V}^{3} / \mathrm{m}^{3}\right)$ of the Russas 'free-standing' orange trees, Russas-CE-Brazil.

Constatou-se alta afinidade entre os clones BRS Russas enxertados sobre os citrandarins 'Indio e San Diego', atribuindo-se qualquer variação amostral às diferentes exigências das copas e do portas-enxertos sob estresses abióticos, aos quais as copas de clones BRS Russas, estariam melhor adaptadas, que os porta-enxertos híbridos.

A variedade laranja 'de Russas' se caracterizou tradicionalmente pela propagação seminal, prática que norteou as primeiras espécies cítricas introduzidas no Brasil, logo após a colonização, durante o século XVI, disseminando-se por todo país em função da facilidade deste método na obtenção de mudas, e que ainda perdura na citricultura em escala familiar no semiárido nordestino (CUNHA SOBRINHO et al., 2013; SOMBRA et al., 2016).

A literatura apresenta, entre outras desvantagens que inviabilizam, ou contraindicam, o cultivo de laranjeiras 'pés francos', na maioria das situações: a alta variabilidade genética e desuniformidade no pomar; o retardamento no início da produção em relação ao das plantas enxertadas, devido à presença da juvenilidade; abundância na formação de espinhos, principalmente no período juvenil; copas vigorosas, com crescimento predominantemente vertical, o que tende a dificultar e onerar os tratos culturais em geral; além de, maior susceptibilidade a diversas doenças disseminadas via solo, em relação a porta-enxertos melhorados geneticamente, tolerantes ou resistentes ao vírus causador da Tristeza (Citrus tristeza virus - CTV), exocorte (Citrus exocortis viroid $-C E V d$ ); xiloporose (Hop stunt viroid - HSVd); nematoides; gomose de Phytophthora spp. (Peronosporales: Pythiaceae); Declínio e Morte Súbita do citros (MSC) (MOREIRA et al., 1954; ALMEIDA; PASSOS, 2011; CUNHA SOBRINHO et al., 2013).

A propagação seminal predominou até a segunda metade do século XIX, período em que se relatam o desenvolvimento da citricultura no semiárido cearense, onde originou-se a laranja 'de Russas', porém, assim como no semiárido, a propagação seminal teve seu declínio já no começo do século seguinte, principalmente devido a proliferação de Phytophthora spp., na Ilha dos Açores em Portugal, quando iniciaram-se aos primeiros estudos do uso de porta-enxertos tolerantes ou resistentes a estes fungos (ANDRADE; MARTINS, 2003).

Já os espanhóis, estiveram entre os primeiros citricultores a observarem que as plantas seminais, ou pés francos, tardavam a superar o período de juvenilidade, e consequentemente, a entrar em produção, além da presença de espinhos, que geralmente lesionavam as frutas e dificultavam os tratos culturais, motivando a adoção da enxertia como principal técnica de propagação de plantas cítricas (ANDRADE; MARTINS, 2003; BARBIERI; STUMPF, 2008).

Permitindo inferir que laranjeiras 'de Russas' pés francos tardam na formação em diâmetro da copa, direcionando seu crescimento verticalmente devido ao alto vigor, enquanto os clones BRS Russas, devido ao processo de enxertia sobre os diferentes porta-enxertos, não apresentam o período juvenil e diante do menor vigor, se desenvolve vegetativamente logo que transplantado, além de produzir precocemente.

A enxertia induz vantagens como: a) precocidade na produção (transferência de maturidade - redução do período juvenil); b) Indução ao nanismo, redução no porte da planta 
(altura) e melhoria na realização dos tratos culturais; c) viabilização do cultivo de espécies ou variedades susceptíveis a problemas fitossanitários e/ou ambientais (estresses bióticos ou abióticos) através do uso de porta-enxertos tolerantes ou resistentes; d) permitir, assegurar ou expandir características desejáveis segregadas por mutações naturais ou induzidas, ou mesmo, controlar segregações indesejáveis; e) preservação e/ou multiplicação de variedades nobres de alta produtividade e características desejáveis (matrizes ou espécimes de alta qualidade - como as laranjeiras 'de Russas' matrizes dos clones BRS Russas); f) permite a renovação de pomares em declínio, através da substituição de plantas indesejáveis em campo (ALMEIDA; PASSOS, 2011; SOMBRA et al., 2016).

Em face a importância, a caracterização botânica de mutações da variedade tradicional 'laranja de Russas', ainda propagados via sementes por alguns pequenos (as) citricultores (as) nos pomares produtivos do semiárido cearense, é fundamental para identificação de matrizes com potencial agronômico, capazes de originar clones nobres, de alta produtividade; resistentes ou tolerantes a artrópodespragas ou doenças; capazes de produzir frutos com características organolépticas atrativas como, por exemplo, coloração ou espessura de casca ou polpa, baixo número de sementes, graus de sólidos solúveis ou acidez mais baixos ou elevados, atendendo diferentes demandas do consumidor de frutas de mesa.

Porém, diante das desvantagens inerentes às plantas 'pés francos', contraindica-se a implantação de pomares com mudas seminais de laranjeiras de Russas, dando-se preferência a combinações copa e porta-enxertos, se possível clones BRS Russas, adaptados as condições edafoclimáticas típicas de semiárido, que vem desenvolvendo-se de forma satisfatória segundo os resultados, além da capacidade de produção de laranjas com potencial de atender a demanda por frutas cítricas de mesa, seja a nível regional ou nacional (PASSOS et al., 2013; SOMBRA et al., 2018).

\section{CONCLUSÕES}

As laranjeiras pés francos da variedade 'De Russas' apresentaram desenvolvimento vegetativo inferior ao dos clones BRS Russas em quase todas as variáveis na fase juvenil, indicando que apesar do vigor das plantas seminais, a ausência da juvenilidade proporcionada pelo uso de mudas enxertadas, associada ao adensamento no espaçamento pode ter favorecido as plantas e proporcionado maior rapidez na formação das copas dos clones em condições semiáridas.

Os clones BRS Russas também demonstraram alta afinidade com os porta-enxertos avaliados, destacando-se o desenvolvimento vegetativo do clone BRS Russas 04 sobre os citrandarins 'Indio e San Diego', além do citrumelo 'Swingle', demonstrando alguma adaptação aos estresses abióticos típicos do semiárido cearense.

As laranjeiras pés francos em período produtivo apresentavam desenvolvimento vigoroso, com predomínio de crescimento vertical nos primeiros anos, porém, ganhando diâmetro e volume de copa nos anos posteriores, quando geralmente dá-se a estabilidade produtiva e constatam-se as produtividades elevadas e retornos econômicos significativos na citricultura tradicional.

\section{AGRADECIMENTOS}

Ao Conselho Nacional de Desenvolvimento Científico e Tecnológico (CNPq) pela concessão de bolsas, à Embrapa
Mandioca e Fruticultura Tropical e à Embrapa Semiárido, à Secretaria de Agricultura de Russas (SEAGRI) e o Núcleo de Pesquisa em Citros (NPCitrus/IFCE).

\section{REFERÊNCIAS}

ALMEIDA, C. O.; PASSOS, O. S. Citricultura brasileira: em busca de novos rumos desafios e oportunidades na região Nordeste. 1. ed. Cruz das Almas: Embrapa Mandioca e Fruticultura Tropical, 2011. 160 p.

ALVARES, C. A.; STAPE, J. L.; SENTELHAS, P. C.; DE MORAES, G.; LEONARDO, J.; SPAROVEK, G. Köppen's climate classification map for Brazil. Meteorologische Zeitschrift, Stuttgart, v. 22, n. 6, p. 711728, 2014. DOI: https://dx.doi.org/10.1127/09412948/2013/0507

ANDRADE, R. A.; MARTINS, A. B. G. Propagação vegetativa de porta-enxertos para citros. Revista Brasileira de Fruticultura, Jaboticabal, v. 25, n. 1, p. 134-136, 2003. DOI: http://dx.doi.org/10.1590/S010029452003000100038

BARBIERI, R. L.; STUMPF, E. R. T. Origem e evolução de plantas cultivadas. Brasília: Embrapa Informação Tecnológica, 2008. 909 p.

CUNHA SOBRINHO, A. P.; MAGALHÃES, A. F. J.; SOUZA, A. S.; PASSOS, O. S.; SOARES FILHO, W. S. Cultura do Citros. Brasília: Embrapa Informação Tecnológica, v. 1, 2013.399 p.

FALLAHI, E.; RODNEY, D. R. Tree size, yield, fruit quality, and leaf mineral nutrient concentration of 'Fairchild' mandarin on six rootstocks. Journal of the American Society for Horticultural Science, Geneva, v. 116, n. 1, p. 2-5, 1991.

IBGE INSTITUTO BRASILEIRO DE GEOGRAFIA E ESTATÍSTICA. Levantamento Sistemático da Produção Agrícola: Pesquisa mensal de previsão e acompanhamento das safras agrícolas no ano civil LSPA. Rio de Janeiro, v. 30, n. 1, p. 1-81. 2017.

LOUREIRO, F. L. C.; SOMBRA, K. E. S.; SILVA, A. C. C.; PASSOS, O. S.; BASTOS, D. C. Avaliação Biométrica de Pomeleiro Star Ruby sob Diferentes Porta-Enxertos no Semiárido do Ceará. Revista Técnico Científica do CREA-PR, Curitiba, v. 1, p. 1-9, 2016.

MOREIRA, S.; COSTA, A. S.; GRANT, T. J. Métodos para identificação e controle da tristeza dos citros. Bragantia, Campinas, v. 13, n. 1, p. 223-236, 1954.

PASSOS, O. S.; SOARES FILHO, W. S.; BARBOSA, C. J.; CUNHA SOBRINHO, A. P. Clones de laranjeira 'DE RUSSAS'. Cruz das Almas: Embrapa Mandioca e Fruticultura, 2013. 4 p. (Folder/Folheto/Cartilha).

PASSOS, O. S.; SOARES FILHO, W. S.; CUNHA SOBRINHO, A. P.; SOUZA, A. S.; SANTOS, L. C.; PEIXOUTO, L. S. Banco ativo de germoplasma de citros da Embrapa Mandioca e Fruticultura. Cruz das Almas: Embrapa Mandioca e Fruticultura, 2016. 6 p. (Folder/Folheto/Cartilha).

RODRIGUES, M. J. S.; LEDO, C. A. S.; GIRARDI, E. A.; ALMEIDA, L. A. H.; SOARES FILHO, W. S. Caracterização de frutos e propagação de porta-enxertos híbridos de Citros em ambiente protegido. Revista Brasileira de Fruticultura, Jaboticabal, v. 37, n. 2, p. 457-470, 2015. DOI: http://dx.doi.org/10.1590/0100$2945-068 / 14$ 
RODRIGUES, M. J. S.; OLIVEIRA, E. R. M.; GIRARDI, E. A.; LEDO, C. A. S.; SOARES FILHO, W. S. Produção de mudas de Citros com diferentes combinações copa e portaenxerto em viveiro protegido. Revista Brasileira de Fruticultura, Jaboticabal, v. 38, n. 1, p. 187-201, 2016. DOI: http://dx.doi.org/10.1590/0100-2945-284/14

SILVA, F. A. S.; AZEVEDO, C. A. V. The Assistat Software Version 7.7 and its use in the analysis of experimental data. African Journal of Agricultural Research, v. 11, n. 39, p. $3733-3740$. 2016. DOI: http://dx.doi.org/10.5897/AJAR2016.11522

SILVA, C. E. F.; DA GAMA, B. M. V.; OLIVEIRA, L. M. T. M.; ARAUJO, L. T.; ARAUJO, M. L.; DE OLIVEIRA JUNIOR, A. M.; SOUZA ABUD, A. K. Uso da laranja lima e seus resíduos no desenvolvimento de novos produtos. Revista Brasileira de Engenharia de Biossistemas, Tupã, v. 10, n. 1, p. 69-96, 2016. DOI: DOI: http://dx.doi.org/10.18011/bioeng2016v10n1p69-96

SOARES, L. A. A.; BRITO, M. E. B.; FERNANDES, P. D.; LIMA, G. S.; SOARES FILHO, W. S.; OLIVEIRA, E. S. Crescimento de combinações copa-porta-enxerto de citros sob estresse hídrico em casa de vegetação. Revista Brasileira de Engenharia Agrícola e Ambiental, Campina Grande, v. 19, n. 3, p. 211-217. 2015. DOI: http://dx.doi.org/10.1590/1807-

1929/agriambi.v19n3p211-217
SOMBRA, K. E. S.; SILVA, A. C. C.; LOUREIRO, F. L. C.; BASTOS, D. C. Citricultura desenvolvida na agricultura de base familiar do município de Russas, Ceará. Cultura Agronômica, Ilha Solteira, v. 25, n. 3, p. 303-316, 2016.

SOMBRA, K. E. S.; SILVA, A. C. C.; LOUREIRO, F. L. C.; UCHÔA, C. N. A Citricultura como Instrumento de Preservação da Agricultura Familiar no Semiárido Cearense, Brasil. Revista de Extensão e Estudos Rurais, Viçosa, v. 7, n. 1, p. 353-372, 2018a. DOI: https://doi.org/10.18540/rever712018353-372

SOMBRA, K. E. S.; LOUREIRO, F. L. C.; COSTA, A. C., SILVA; SILVA, M. P.; PASSOS, O. S.; BASTOS, D. C. Avaliação biométrica de tangelo Page e pomelo Flame sobre diferentes porta-enxertos no semiárido do Ceará, Brasil. Citrus Research \& Technology, Cordeirópolis, v. 39, e-1038, 2018b. http://dx.doi.org/10.4322/crt.16418

USDA_UNITED STATES DEPARTMENT OF AGRICULTURE. Citrus: World markets and trade (July 2017). Washington, DC: Foreign Agricultural Service, 2017a. 9 p.

USDA. United States Department of Agriculture. Brazil Citrus Semi-annual - GAIN Reports. Washington, DC: Foreign Agricultural Service, 2017b. 11 p. 